\title{
New Instructional Models for Building Effective Curricula on Cloud Computing Technologies and Engineering
}

\author{
${ }^{1}$ Yuri Demchenko, ${ }^{2}$ David Bernstein, ${ }^{1}$ Adam Belloum, ${ }^{1}$ Ana Oprescu, ${ }^{3}$ Tomasz W. Wlodarczyk, ${ }^{1}$ Cees de Laat \\ ${ }^{1}$ University of Amsterdam \\ System and Network Engineering Group \\ Amsterdam, The Netherlands \\ e-mail: \{y.demchenko, a.s.z.belloum, a.m.oprescu, delaat\}@uva.nl \\ ${ }^{2}$ Cloud Strategy Partners, LLC \\ Silicon Valley, California, USA \\ e-mail: david@cloudstrategypartners.com \\ ${ }^{3}$ University of Stavanger \\ Stavanger, Norway \\ e-mail: tomasz.w.wlodarczyk@uis.no
}

\begin{abstract}
This paper presents ongoing work to develop advanced education and training course on the Cloud Computing technologies foundation and engineering by a cooperating group of universities and the professional education partners. The central part of proposed approach is the Common Body of Knowledge in Cloud Computing (CBKCC) that defines the professional level of knowledge in the selected domain and allows consistent curricula structuring and profiling. The paper presents the structure of the course and explains the principles used for developing course materials, such as Bloom's Taxonomy applied for technical education, and andragogy instructional model for professional education and training. The paper explains the importance of using the strong technical foundation to build the course materials that can address interests of different categories of stakeholders and roles/responsibilities in the Cloud Computing services provisioning and operation. The paper provides a short description of summary of the used Cloud Computing related architecture concepts and models that allow consistent mapping between CBK-CC, stakeholder roles/responsibilities and required skills. The paper refers to the ongoing development of the educational course on Cloud Computing at the University of Amsterdam, University of Stavanger and provides suggestions for building advanced online training course for IT professionals.
\end{abstract}

Keywords- Education and Training on Cloud Computing Technologies; Instructional methodology; Common Body of Knowledge; Bloom's Taxonomy; Andragogy; Multi-layer Cloud Services Model; Intercloud Architcture Framework.

\section{INTRODUCTION}

Cloud Computing [1,2] is an important new paradigm in the area of Information Technology (IT) that currently provides a (common) basis for a new wave of the technology development such as Big Data and pushing further mobility based pervasive computing concept. It represents what most industry experts characterize as computing architecture as important and distinctive as "Mainframe", "Client Server", and "Internet". Services such as email, Internet search, social network, Internet video, conferencing and sharing technologies - which are becoming no less than vital for our everyday lives - are moving to cloud platforms, or have already become cloud based. It is also the technology behind datacenter consolidation, outsourcing and hosting, and next generation supercomputing.

In general, Cloud Computing technologies are emerging as a common platform for provisioning general infrastructure services and domain specific applications on-demand that can be designed to support user specific applications, processes and workflows and dynamically created user groups.

This paper presents a new and comprehensive approach to designing a Cloud Computing curricula to address needs of the different professional groups in Cloud Computing. The proposed curriculum intends to provide an overview and analysis of the existing cloud architecture models, cloud industry standardisation, existing cloud middleware platforms and major cloud provider platforms. To ensure the consistency of technical content, the course uses the two generic architecture components the Cloud Services Model (CSM) and the InterCloud Architecture Framework (ICAF) $[4,5]$ that are defined according to the recent recommendations by ITU-T, IEEE, IETF and OGF standardisation bodies, and industry best practices on cloud based infrastructure services design and management.

All main topics of the course contain the main/foundational part that provides the basic information sufficient for understanding and using specific clouds functions, and the advanced part that provide additional information for deep understanding and advanced use of the technology sufficient for cloud services and applications development and implementation. An important part of the course is the security and policy management issues in clouds, including provider security, user applications security, federated identity management, compliance and certification.

Cloud Computing embodies advances in computing, networking, and storage technologies which individually and collectively include major hardware and software breakthroughs. These include compute virtualization, distributed and replicated storage, and software based networking. In its own turn, Cloud Computing motivates further technology advancement and changes how the modern IT infrastructure is built and evolves. There is a strong demand for specialists with strong technical 
background and deep knowledge of the Cloud Computing technologies. However, there is no widely available Cloud Computing training and professional education as well as there is no common approach to building professional level Cloud Computing curricula.

Hardware and software engineers are increasingly searching for more education on these new technologies, both out of technical curiosity and out of necessity for their careers. Universities recognize that Cloud Computing is a mandatory technology and knowledge for the future emerging IT technologies such as Big Data and Data Intensive Science. The presented paper discusses the general instructional approach to creating/building comprehensive curriculum, covering the "A-Z" of Cloud Computing at the level sufficient for informed use of the basic cloud technologies, cloud services development and further professional development.

The curriculum design outlined here presents a complete course in Cloud Computing, aimed at the technical product planner, consultant, or engineer. The modules are structured along the lines of common industry terms and features of Cloud Computing, so that the student with a passing knowledge of the subject will be able to instantly recognize the subject matter of the course.

The remainder of the paper is organized as follows. Section II explains need for a new approach in Cloud Computing education and training and outlines the main properties of the professional level of knowledge. Section III introduces the Common Body of Knowledge in Cloud Computing (CBK-CC) and explains inter-relations between its elements. Section IV provides short overview of the Cloud Services Model (CSM) and the InterCloud Architecture Framework (ICAF) including also the main stakeholders definition in cloud service provisioning and operation that jointly provide an architectural and technical basis for CBK$\mathrm{CC}$ and the course design. Section V describes mapping between CSM and course profiles reflecting required knowledge and skills for the main stakeholder activities. Section VI and VII explain correspondingly how the new advanced instructional methodologies such as Bloom's taxonomy and Andragogy are used to improve the proposed course delivery and learning outcome. Section VIII discusses few examples of the education and training programs development in which the authors are involved, and the paper concludes with the summary and future developments in section IX.

\section{DEMAND OF TIME: INNOVATIVE EDUCATIONAL APPROACH}

Having delivered many courses in computer science, networking, Grid Computing, Security Engineering, and related, the authors have realized that the usual educational approaches do not serve that well in providing advanced curriculum to professionals on new emerging technologies. It has become clear that some very particular techniques need to be utilized in order to provide a deeper, more efficient, and more enjoyable learning experience.

So we will utilize these principles in developing a new type of curriculum. In the following sections we will explain the new instructional methods and education approaches we found effective for such new emerging technology domains as Cloud Computing.

\section{A. Principles of Professional Education}

The discussed here curriculum seeks to provide knowledge both in Cloud Computing as a new technology and the background technologies which go into Cloud. Our objective is to empower future professionals with the ability to develop new knowledge and build stronger expertise in Cloud, and also to prepare a basis for new emerging technologies such as Big Data.

As a basis, a professional education needs to provide for the professional level of knowledge that can be outlined as follows:

1) Knowing basic concepts and major application areas

2) Knowing similar concepts (and concepts inter-relation) and alternatives, as well as application specific areas

3) Knowing basic technologies and their relation to basic concepts.

Beyond this, a professional needs additional "dimensions" of awareness and critical thinking:

4) Knowing authoritative (and not authoritative) sources of information and how to evaluate quality of information

5) Ability to work with standards (what is not an easy source of information)

6) Ability to critically evaluate and filter some inconsistent information, e.g. popular sites like Wikipedia and similar, blogs, etc.

7) Critically evaluate vendors' information which is sometimes biased and/or doesn't provide enough background information

Cloud computing is still a new technology today, but it is becoming a common preferred base/platform for all current and future developments. Professionals want to become an "expert" in this new area. They need both a general professional knowledge and understanding of the main development areas, as well as practical development and experience with activities such as projects, writing reports, technical documents, and following and contributing to standardization.

As the result of the proposed curriculum, professionals should become "cloud aware", armed with "cloud powered design" and capable of "cloud powered analysis and thinking".

\section{COMMON Body OF KNOWLedge IN Cloud COMPUTING (CBK-CC)}

CBK refers to several domains or operational categories into which Cloud Computing theory and practices breaks down. It is like a Blueprint or Framework which students can see how things fit together.

For Cloud Computing, we identify the following CBK elements:

1. Cloud Computing Architectures, service and deployment models

2. Cloud Computing platforms, software/middleware and API's

3. Cloud Services Engineering, Cloud aware Services Design

4. Virtualization technologies (Compute, Storage, Network)

5. Computer Networks, Software Defined Networks (SDN)

6. Service Computing, Web Services and Service Oriented Architecture (SOA)

7. Computing models: Grid, Distributed, Cluster Computing

8. Security Architecture and Models, Operational Security 
9. IT Service Management, Business Continuity Planning (BCP)

10. Business and Operational Models, Compliance, Assurance, Certification

Figure 1 shows the $2 \mathrm{D}$ relations between the $\mathrm{CKB}$ components and illustrates their mapping to the required skills and course subsets or profiles. Accordingly, the core Cloud Computing Fundamentals course includes such topics as Cloud Computing Architectures, service and deployment models; Cloud Computing platforms, software/middleware and API's; Cloud aware Services Design; Compute and Storage Virtualization; Web Services and Service Oriented Architecture (SOA); Cloud Security, Operational Security, Identity management; Business and Operational Models, Compliance, Assurance.

The Cloud Computing Fundamentals provide sufficient basis for performing the main professional tasks and further independent professional development. Different groups of the cloud professionals and specialists can extend their education and training with the specific knowledge and skills according to the CBK-CC.

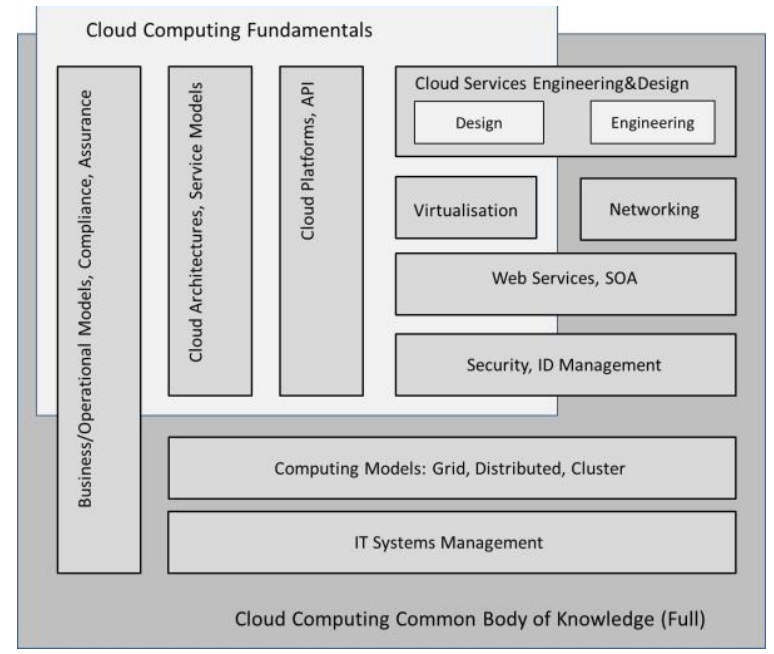

Figure 1. CKB-CC elements inter-relation and mapping to course profiles.

\section{Cloud COMPUTING ARCHITECTURE FOUNDATION}

The key component of the consistent course organization is the strong conceptual and architectural background used for creating logical structure between topics/modules, learning targets and their relation to practical business and operational needs. Well defined architectural background provides a basis for consistent vocabulary and stakeholder roles, actions and interest definition.

The course provides overview of the main Cloud Computing architectures proposed by different standardization bodies NIST [1, 2], IEEE [5], ITU-T [6], IETF [7], ODCA [8], comprehensive architectures defined by leading industry actors such as Amazon AWS [9], Microsoft Azure [10], IBM [11], and proposes consolidated architecture view comprising of the Cloud Services Model (CSM) and Intercloud Architecture Framework (ICAF) [3] as explained below.

\section{A. Cloud Service Model}

Figure 2 illustrates the proposed multi-layer CSM definition that reflects the main functional layers and related functional components in a typical cloud infrastructure. It combines the basic cloud service models IaaS, PaaS, SaaS into one Cloud Services Layer that typically expose standard based interfaces to user applications or services but can use proprietary interfaces to the underlying provider platform. In this respect, the proposed model provides a basis for the interlayer interfaces definition and mapping.

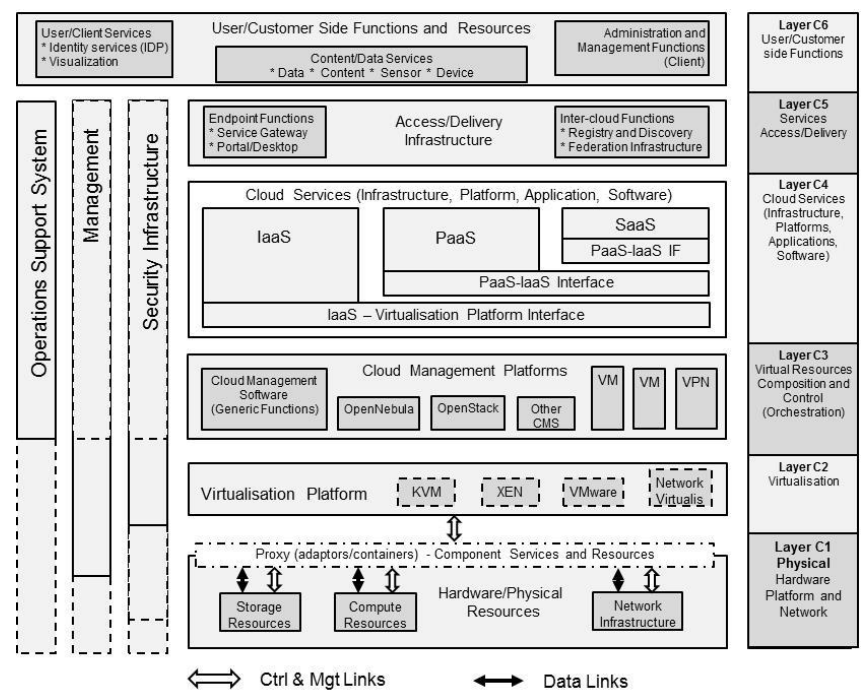

Figure 2. Reference Multilayer Cloud Services Model (CSM).

In the proposed CSM the following layers are defined including user client or application at the top (numbering from bottom up, see Fig. 2):

(C6) User/customer side resources and services located in and provided by the customer's enterprise or campus network to support their integration with the cloud based infrastructure; these may include identity management, infrastructure administration, data services and visualization. (C5) Access/Delivery infrastructure hosting components and functions to provide access to cloud services/resources and interconnect multiple cloud domains; cloud federation services are located at this layer.

(C4) Cloud services layer that may include different type of cloud services IaaS, PaaS, SaaS that are exposed to upper customer faced layer via standard interfaces while potentially using non-standard internal and lower facing interfaces.

(C3) Cloud virtual resources composition and orchestration layer that is represented by the Cloud Management Software (including such platforms as OpenNebula, OpenStack, or others) and may include additional services for combined inter-cloud infrastructure composition and orchestration.

(C2) Cloud virtualisation layer (e.g. represented by VMware, Xen or KVM as virtualisation platforms; or using custom virtualisation platform for non-computer resources).

(C1) Physical platform (PC hardware, storage, network, and network infrastructure); to interface with the virtualisation layer the physical resources layer may provide special adaptors' functionality.

The three vertical planes or cross-layer infrastructures are defined to group related functionality in all CSM layers:

- Control and Management Plane

- Operations Support System

- Security Infrastructure. 
The CSM includes all the major components of the general cloud services design/functional components residing at the Cloud Service Provider (CSP) and those functional components constituting the inter-cloud access and services delivery infrastructure including also user client and applications.

\section{B. Intercloud Architecture Framework (ICAF)}

The proposed Intercloud Architecture Framework (ICAF) addresses the interoperability and integration issues in the current and emerging heterogeneous multi-domain and multi-provider clouds that could host modern and future critical enterprise and e-Science infrastructures and applications, including integration and interoperability with legacy campus/enterprise infrastructure.

The ICAF includes the following components that separate all functions related to the cloud services design, control, management and operations into "orthogonal" groups:

(1) Multilayer Cloud Services Model (CSM) (explained above) for vertical cloud services interaction, integration and compatibility that defines both relations between cloud service models (such as IaaS, PaaS, SaaS) and other required functional layers and components of the general cloud based services infrastructure, including also the Access and Delivery Infrastructure layer.

(2) Intercloud Control and Management Plane (ICCMP) for Intercloud applications/infrastructure control and management, including inter-applications signaling, synchronization and session management, configuration, monitoring, run time infrastructure optimization including VM migration, resources scaling, and jobs/objects routing.

(3) Intercloud Federation Framework (ICFF) to allow independent clouds and related infrastructure components federation of independently managed cloud based infrastructure components belonging to different cloud providers and/or administrative domains; this should support federation at the level of services, business applications, semantics, and namespaces, assuming necessary gateway or federation services.

(4) Intercloud Operation and Management Framework (ICOMF) which includes functionalities to support multiprovider infrastructure operation, including business workflow, SLA management and accounting. ICOF defines the main stakeholders, actors and their roles and relations in sense of resources ownership, operation, and management. ICOF requires support from and interacts with both ICCMP and ICFF.

(5) Intercloud Security Framework (ICSF) that provides a basis for secure operation of all components of the Intercloud infrastructure, including secure operation of the cloud federations. In this respect ICSF should provide a basis for integration of the security services between different CSM layers and all participating cloud service providers.

\section{Main/Top Level Stakeholders}

To be effective and market successful, education and training program should target the main stakeholder groups and their interests in the cloud services delivery model, which are typically related to the ICOMF.
We define the following main actors and roles adopting the Resource-Ownership-Role-Action (RORA) model proposed in $[12,13]$ and referring to other service delivery and operations frameworks such as defined by NIST [2, 14] and TMF Service Delivery Framework (SDF) [15]:

- Cloud Service Provider (CSP) as entity providing cloud based services to customers, on their request and based on the business agreement that is expressed as Service Level Agreement (SLA). It is important to admit specifics of business relations in clouds due to the fact that majority of cloud services are self-serviced and are governed under general or individualized SLA.

- Cloud Broker is an entity that may play a role of the third party in offering cloud service adding value of negotiating with many CSPs or customer groups and in some cases managing complex multi-provider services.

- Cloud Service Operator is a new emerging role of the company that provides a value added service of integrating services from multiple cloud providers, delivering them to the customer and operating the resulting infrastructure.

- Cloud Service Integrator is a role provided by the third party IT company to design and deploy the cloud services infrastructure that matches the customer requirements; this role includes detailed customer needs and requirements study and proposal of the optimized cloud based infrastructure, including also need for customer IT infrastructure redesign.

- Cloud Auditor is a party that can conduct independent assessment of cloud services, information system operations, performance and security of the cloud implementation.

- Cloud Carrier is an intermediary that provides connectivity and transport of cloud services from Cloud Providers to Cloud Consumers; Cloud Carrier services is typically provided by a telecom company.

- Customer (like enterprise or university) is entity that request service from and has contractual relations with the CSP; customer does cloud services management themselves or outsource it to the third party.

- User is an end-user consuming cloud based services; in cloud services provisioning.

Addressing education and training skills requirements for the major industry stakeholders and roles is a condition for the course acceptance by the industry and wide professional community.

\section{MAPPING Stakeholder DOMAINS AND COURSE COMPONENTS}

Mapping between CSM, major stakeholder service domains, and required knowledge (or competencies) is an important next step in building consistent curricula. This is also important for effective course profiling for the target group of students or trainees that can be used for defining the course modules that will be delivered to different groups of attendees/subscribers/listeners.

We understand the difference for students where we can just deliver a complete program, and an advance professional training where the educator needs to more specifically target the specific interests of trainees. 


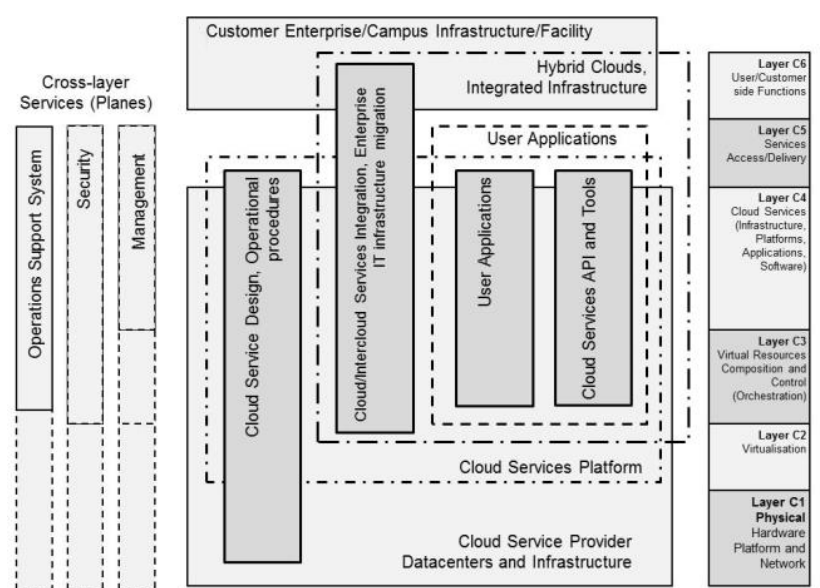

Figure 3. Mapping between Cloud Services Model (CSM), major stakeholder service domains, and required knowledge/skills.

The following major course profiles/modules can be defined:

- User Applications module targets a wide group of the cloud users that need to use cloud services provided by the public cloud service providers or private clouds run by enterprise. This user group should have also skills to develop their own cloud applications using one of the available cloud management platforms in the private or public cloud.

- Cloud Service Platforms module targets both cloud services developers (using available cloud services platforms) and technical personnel of the cloud service providers that both require deeper knowledge of the cloud services design, software, virtualization platforms, operations and business processes. It is understood that the future employees will require special training by their employer company, but the course will provide all the basic knowledge for their quick mastering of the provider or enterprise specific technologies.

- Cloud/Intercloud Services Integration, Hybrid Clouds course module targets wide range of businesses (including cloud service brokers, integrators, operators, and also cloud carriers) that provide cloud integration services for enterprises and large cooperative projects. This module includes detailed analysis of the customer requirements, workflow and current IT infrastructure study and proposing the optimized cloud based infrastructure.

\section{USING BLOOM'S TAXONOMY FOR DEFINING} LEARNING TARGETS AND OUTCOME

\section{A. Bloom's Taxonomy Summary and Example}

Bloom's Taxonomy is a basis for defining learning targets and modules outcome $[16,17,18,19]$. It sequences learning into a number of cognitive levels defining learning outcome:

\section{Knowledge}

Exhibit memory of previously learned materials by recalling facts, terms, basic concepts and answers

- Knowledge of specifics - terminology, specific facts

- Knowledge of ways and means of dealing with specifics - conventions, trends and sequences, classifications and categories, criteria, methodology
- Knowledge of the universals and abstractions in a field principles and generalizations, theories and structures Questions like: What are the main benefits of outsourcing company's IT services to cloud?

\section{Comprehension}

Demonstrate understanding of facts and ideas by organizing, comparing, translating, interpreting, describing, and stating the main ideas

- Translation, Interpretation, Extrapolation

Questions like: Compare the business and operational models of private clouds and hybrid clouds.

\section{Application}

Using new knowledge, solve problems in new situations by applying acquired knowledge, facts, techniques and rules in a different way

Questions like: Which cloud service model is best suited for medium size software development company, and why?

Analysis

Examine and break information into parts by identifying motives or causes. Make inferences and find evidence to support generalizations

- Analysis of elements, relationships, organizational principles

Questions like: What cloud services are needed to support typical business processes of a web trading company, give suggestions how these services can be implemented with PaaS or IaaS clouds? Provide references to support your statements.

Synthesis

Compile information together in a different way by combining elements in a new pattern or proposing alternative solutions

- Production of a unique communication, a plan, or proposed set of operations, derivation of a set of abstract relations

Questions like: Describe the main steps and tasks for migrating IT services of an example company to clouds, what services and data can be moved to clouds and which will remain at the enterprise premises.

\section{Evaluation}

Present and defend opinions by making judgments about information, validity of ideas or quality of work based on a set of criteria

- Judgments in terms of internal evidence or external criteria

Questions like: Do you think that cloudification of the enterprise infrastructure creates benefits for enterprises, short term and long term?

\section{B. Mapping Course modules to Bloom's Taxonomy}

Figure 4 provides mapping Bloom's Taxonomy to professional activity domains and to required knowledge and skills:

- Knowledge:

Perform standard tasks, use standard API and Guidelines

- Comprehension:

Create own complex applications using standard API (simple engineering)

- Application:

Integrate different systems/components, e.g. provider and enterprise infrastructure

- Analysis:

Extend existing services, design new services 
- $\quad$ Synthesis

and

Evaluation:

Develop new architecture and models, platforms and infrastructures

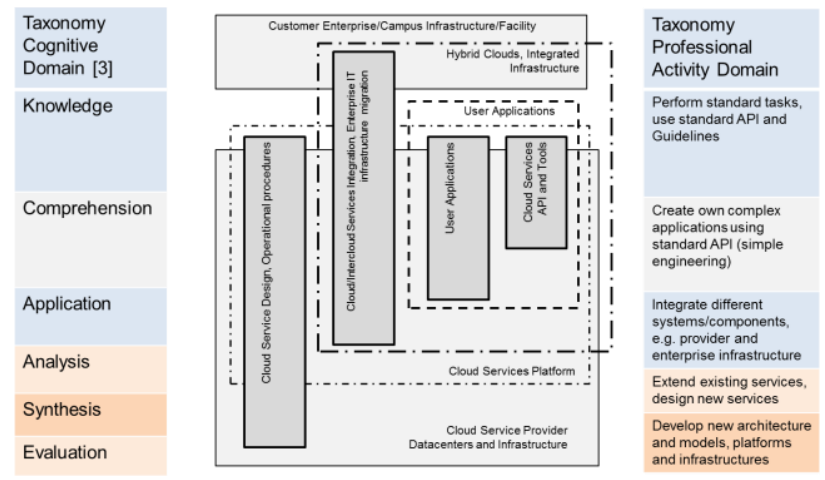

Figure 4. Mapping Bloom's Taxonomy and professional activity domains to required knowledge/skills

The proposed mapping is actually related to the whole body of knowledge that constitutes the expert knowledge in Cloud Computing. However in general, Bloom's Taxonomy can be applied to any piece of a curriculum with the correspondingly defined learning objectives.

\section{Methodology OF AndRAgOgy (VS. PedAgOGY)}

Andragogy consists of learning strategies focused on adults $[20,21,22]$. It is often interpreted as the process of engaging adult learners with the structure of learning experience. The andragogy principles are highly elated to professional education that typically has high motivation factor. It can be applied in on-campus and on-line education. Developed by American educator Malcolm Knowles [20], he stated six assumptions related to motivation of adult learning:

1. Adults need to know the reason for learning something (Need to Know)

2. Experience (including error) provides the basis for learning activities (Foundation)

3. Adults need to be responsible for their decisions on education; involvement in the planning and evaluation of their instruction (Self-concept)

4. Adults are most interested in learning subjects having immediate relevance to their work and/or personal lives (Readiness).

5. Adult learning is problem-centered rather than contentoriented (Orientation)

6. Adults respond better to internal versus external motivators (Motivation)

Andragogy provides effective approach to online higher education that is offered by high ranked online universities such as Laureate Online Higher Education (LOHE) [23, 24], the online education branch of the Liverpool University. The LOHE provides fully online teaching/education environment based on customized Blackboard platform. The following activities and education forms are used in Computer Science online master education:

- The module consists of 8 weekly seminars that includes 2 Discussion Questions (DQ), Hands-in Assignment (HA, or homework) and project assignment.

- Each seminar is provided with the Lecture Notes and textbook reading assignment. There is no recorded or synchronous lectures, what makes also possible education delivery to countries and to students with low Internet connectivity.

- Discussion questions and asynchronous discussion are the main form of educational activity. DQ answers are submitted to the discussion forum and the students are required to contribute to the discussion. The students benefit from the knowledge and experience sharing during discussion and learn how to defend own answer. Instructor plays a role of moderator and the students' knowledge and activities assessor.

- Discussion questions are designed in such a way that to stimulate the students higher cognitive activities starting from the basic literature search to analyzing and evaluating collected information and further to making concise knowledge synthesis and their application to problem solving.

- Criteria for assessing the students' knowledge and learning outcome are based on the Bloom's Taxonomy and require some experience from the instructor.

We discuss further application of the andragogy principles to online self-education courses in the following section.

\section{IMPLEMENTATION STATUS AND SUGGESTIONS}

\section{A. SOA and Cloud Computing Course for Master students at the University of Amsterdam}

The SOA and Cloud Computing course is being developed for the Master Computer Science full-time students and counts for 6 credits. The course runs 2 months and has 8 contact hours per week of which 4 hours are lectures and 2 lab hours.

The course also includes the final projects to design cloud based application or service to support a selected business process or scientific workflow using one of the available cloud platforms: local cloud testbed, Amazon AWS, or Microsoft Azure. The lab assignments include the basic hands-on tasks to learn how to work with virtualisation and cloud platforms both provided by the local cloud testbed and two major cloud platforms by Amazon AWS and Microsoft Azure, what together provides a basis for the practical project realization.

The course is built in such a way to facilitate the students deeper study/research into the subject of Cloud Computing and related problems, improve their skill in working with professional and scientific literature, facilitate collaborative learning and actively use their knowledge in the discussion.

To achieve this the course employs two types of selfstudy activities: (1) discussion of the selected papers in the classroom (also called "reading club") that is done weekly and requires submitting two short summaries of the paper before discussion and after discussion where the student is required demonstrate their understanding of the paper and its relation to the weekly lecture topic; and (2) literature based research on individually selected topics that must be resulted in a short essay and its presentation during the special seminar session that takes place either at the end of course or discussion of the selected papers in the classroom. The proposed list of research topics includes more than 30 topics covering all aspects of Cloud Computing and cloud services design; each topic is provided with the initial list of 2-3 
references and the students are required to find other few authoritative references.

The course will use periodical (weekly or by group of topics) knowledge testing with the test developed to assess at least first three Bloom's taxonomy cognitive levels: evaluation, synthesis and analysis, that will employ both multiple choice questions and questions requiring textual answer. The literature research and final project are the key components to achieve the higher level of knowledge in the Cloud Computing.

Important part of the university education is developing research skills that includes formulating a research problem, studying and critically evaluating scientific and technical literature.

The course will also invite a few guest lectures that will be given by industry experts and cooperating industry partners.

\section{B. Data Intensive Systems Course at the University of Stavanger and Purdue University}

Data Intensive Systems course is being developed between Purdue University, AMD Research and University of Stavager for Master or early graduate level students in science and engineering with primary focus on Computer Science student (or Computer Engineering in American nomenclature). The course runs for a full semester (ca. 3 months) and has 10 contact hours per week, of which 4 are lectures, 3 labs and 3 project dedicated office hours

Labs and project are focused on hands-on programming based on the Hadoop platform hosted by one of the universities specifically for the purpose of the course. In addition the course includes a self-study component where each student has to prepare a short presentation on assigned cutting edge technology, which is not part of the main body of the course.

Main course objective are:

- design, construct, test, and benchmark a small data processing cluster

- understand algorithmic complexity

- use functional programming concept to describe data dependencies for program parallization

- describe the concepts and performance of distributed file systems

- $\quad$ understand Hadoop job tracker, task tracker, scheduling issues, communications, and resource management

- construct programs based on MapReduce paradigm for typical algorithmic problems

- understand the differences between RDBMS, data warehouse, unstructured big data, and keyed files.

- understand reliability theory and apply it to design a data intensive system for given reliability

Teaching modules are constructed in correspondence with the above objectives with focus on balance between engineering skills and proper theoretical background.

The course has 4 elements of knowledge testing: final exam, self-study work, weekly laboratory sessions and final project. Final exam and project test students across all levels of Bloom's Taxonomy, while self-study and particular labs tackle chosen levels separately.

Lecturers will deliver the course simultaneously to students at University of Stavanger and Purdue University from both places and including also AMD Research. Despite this delivery method the course will not be in fact an online course. This is mostly due to limitations of the laboratory part. We have considered using a Cloud platform e.g. Amazon AWS; however, limitations of this platform regarding data locality and network performance have temporarily ruled it out. It is possible that these problems could be solved in future opening a possibility for an online course or even MOOC.

Despite these limitations we are already looking into extending the course to a few more institution in the coming years.

\section{Advanced on-line training course for professional IT specialists}

The demand for online self-study and self-training courses is growing and the authors are considering designing an advanced training course for IT professionals.

When applying discussed above techniques, in particular andragogy and Bloom's Taxonomy to online self-education and training, the following specifics will apply:

- Less time to be devoted by trainee; estimated 1 hour per lesson, maximum 3 lessons per course

- Requires the course workflow to be maximum automated, especially if coupled with certification or precertification

- Requires guided activities while allowing self-directed course selection

- Provide course consistency in sense of style, presentation/graphics, etc

- Combined with the Bloom's taxonomy, provide understood targets and outcomes

- Knowledge control questionnaires at the end of lessons or topics.

The course must be designed in such a way that to build a consistent/advanced knowledge foundation and to prepare the listeners for a concise and advanced use of the cloud technologies and tools for emerging technologies such as Big Data, data analytics and new emerging business paradigms motivated by increasing use of the smart mobile devices.

The curriculum design must be more specifically aimed at the main stakeholders to reflect typical roles or functions such as technical product planner, consultant, or engineer. The modules must be structured along the lines of common industry terms and features of Cloud Computing, so that the student with a passing knowledge of the subject will be able to instantly recognize the subject matter of the course.

The online curriculum should have a pre-requisite of having basic or common knowledge in Cloud Computing and provide materials at Intermediate, Advanced, and Expert levels. The Intermediate and Advanced lessons constitute the Cloud Computing fundamentals and aimed for IT decision makers and informed users:

- all concepts are explained, clouds opportunities are demonstrated, general use cases are analysed, examples of use are provided

- general security issues in clouds are explained.

"Advanced" parts are intended for engineers/practitioners developing and operating cloud services, and doing integration/consulting work. 
The online course materials must be developed with high granularity to allow easy course composition for the target groups of trainees and also designing individual profiles.

\section{CONLUSION AND Future DEVELOPMENTS}

This paper presents a new approach to developing the advanced education and training courses on Cloud Computing technologies that can be profiled both for full time university students and for acting IT professionals.

The proposed approach to curriculum development provides the following benefits and possibilities:

- A wider scope of technologies and important topics covered both in cloud Intermediate, Advanced, and Expert, including materials for developers

- A stronger base on standardization and best practices right up to expert knowledge at each level, from manager to developer

- Is intended to achieve level of known certification programs in Cloud Computing

- Has selected topics and level of coverage based on the authors' experience and experience of the cooperating professional education partners, what is essential in mastering cloud technologies from initial involvement to future professional work and cloud applications development.

When successfully tested the course materials and obtained experience can be re-used for new instructional materials developments such as emerging Big Data and Data Intensive Science and Technologies such as falls into the Research Data Alliance (RDA) Interest Group on Educations and Skills Development activity [25, 26]. However, as with the presented Cloud Computing curriculum the prerequisite for such a new course must be a well defined Common Knowledge Base of the target professional education domain and corresponding architecture framework.

\section{REFERENCES}

[1] NIST SP 800-145, "A NIST definition of cloud computing", [online] http://csrc.nist.gov/publications/nistpubs/800-145/SP800-145.pdf

[2] NIST SP 500-292, Cloud Computing Reference Architecture, v1.0. [Online] http://collaborate.nist.gov/twiki-cloudcomputing/pub/CloudComputing/ReferenceArchitectureTaxonomy/NI ST_SP_500-292_-_090611.pdf

[3] Demchenko, Y., M. Makkes, R.Strijkers, C.Ngo, C. de Laat, Intercloud Architecture Framework for Heterogeneous Multi-Provider Cloud based Infrastructure Services Provisioning, The International Journal of Next-Generation Computing (IJNGC), Volume 4, Issue 2, July 2013

[4] Bernstein, D., Ludvigson, E., Sankar, K., Diamond, S., Morrow, M., Blueprint for the Intercloud - Protocols and Formats for Cloud Computing Interoperability. In Internet and Web Applications and Services, 2009. ICIW '09. Fourth International Conference on, 24-28 May 2009, Venice, Italy.

[5] IEEE P2302 - Standard for Intercloud Interoperability and Federation (SIIF). [online] http://standards.ieee.org/develop/project/2302.html

[6] FG Cloud Technical Report (Part 11 to 7). [online] http://www.itu.int/en/ITU-T/focusgroups/cloud/Documents/FG-coudtechnical-report.zip
[7] Cloud Reference Framework. Internet-Draft, version 0.5, July 3, 2013 [online] http://www.ietf.org/id/draft-khasnabish-cloud-referenceframework-05.txt

[8] Open Data Center Alliance (ODCA) http://www.opendatacenteralliance.org/

[9] Amazon AWS Architecture Center. [online] http://aws.amazon.com/architecture/

[10] Microsoft Azure Architecture. http://www.windowsazure.com/enus/develop/net/architecture/

[11] Cloud Computing Reference Architecture 2.0, IBM CC RA team. [online] https://share.confex.com/share/117/webprogram/Handout/ Session9261/CCRA_2.0.pdf

[12] García-Espín, J. A., J. Ferrer Riera, S. Figuerola and Ester LópezA Multi-tenancy Model Based on Resource Capabilities and Ownership for Infrastructure Management. Proc. The 4th IEEE Conf. on Cloud Computing Technologies and Science (CloudCom2012), 3 - 6 December 2012, Taipei, Taiwan. IEEE Catalog Number: CFP12CLUUSB. ISBN: 978-1-4673-4509-5

[13] Makkes, Marc, Canh Ngo, Yuri Demchenko, Rudolf Strijkers, Robert Meijer, Cees de Laat, Defining Intercloud Federation Framework for Multi-provider Cloud Services Integration, The Fourth International Conference on Cloud Computing, GRIDs, and Virtualization (CLOUD COMPUTING 2013), May 27 - June 1, 2013,Valencia, Spain.

[14] NIST SP 500-299: NIST Cloud Computing Security Reference Architecture. [online] NIST SP 500-299: NIST Cloud Computing Security Reference Architecture

[15] TR139, Service Delivery Framework (SDF) Overview, Release 2.0. [online] http://www.tmforum.org/TechnicalReports/ TR139ServiceDelivery/34303/article.html

[16] Anderson, L.W. (Ed.), Krathwohl, D.R. (Ed.), Airasian, P.W., Cruikshank, K.A., Mayer, R.E., Pintrich, P.R., Raths, J., \& Wittrock, M.C. (2001). A taxonomy for learning, teaching, and assessing: A revision of Bloom's Taxonomy of Educational Objectives (Complete edition). New York: Longman

[17] Bloom's Taxonomy. [onine] https://en.wikipedia.org/wiki/Bloom's_Taxonomy

[18] Bloom's taxonomy: the 21st century version. [online] http://www.educatorstechnology.com/2011/09/blooms-taxonomy21 st-century-version.html

[19] Learning Domains or Bloom's Taxonomy, by Don Clark. [online] http://www.nwlink.com/ donclark/hrd/bloom.html

[20] Knowles, M.S., The Modern Practice of Adult Education: From Pedagogy to Andragogy, March 1988, ISBN-10: 0842822135

[21] Delahaye, B.L., D.C. Limerick, G. Hearn, The relationship between andragogical and pedagogical orientations and the implications for adult learning - Adult Education Quarterly, 44(4): 187-200. Sage Publications, 1994

[22] Merriam, S.B., Andragogy and Self-Directed Learning: Pillars of Adult Learning Theory

[23] Laureate Online Education. [online] http://www.university-liverpoolonline.com/online-learning/overview

[24] Hvorecky, J., Applying the SECI Model and Bloom's Taxonomy to the Preparation of Knowledge Management Specialists. Proc. The Knowledge management and Enterprise Solutions Conference. [online]

http://www.cutn.sk/Library/proceedings/km_2012/PDF\%20FILES/Hv orecky.pdf

[25] Research Data Allience. [onlie] https://rd-alliance.org/node

[26] BoF "Education and training in Data Intensive Science", Research Data Alliance (RDA) meeting, 18-20 March 2013, Gothenburg, Sweden. [online] http://www.uazone.org/demch/presentations/ rda2013-gothenburg-bof-education-skills-v02.pdf 